\title{
A Comparison of Nitroglycerine, Bisphosphonates and their Combination in the Management of Post Menopausal Osteoporosis
}

\author{
Raheeb Ahmad Shah ${ }^{1}$ \\ (MBBS, MS) National Health Mission, J \& K, India
}

\begin{abstract}
The incidence of osteoporosis is rising in post-menopausal women and so is the incidence of fractures. A large number of drugs like calcium, vitamin $D$, bisphosphonates, nitroglycerine etc. are available for osteoporosis. In this study we will compare the effect of Nitroglycerine, Bisphosphonates, and Nitroglycerine and Bisphosphonates (combined) on BMD. The primary outcome measure was BMD estimation at 12 months which was measured as a percentage of the baseline study at the start of study. 88 patients were randomised into three groups and were followed for a period of one year. Baseline and yearly BMD was measured and expressed as a percentage of baseline BMD. It was found out that Alendronate is similar to Nitroglycerine with regards to increase in BMD $(p>0.05)$. However the percentage increase in BMD in combination group was statistically significant as compared to bisphosphonate group or nitroglycerine group $(p<0.01)$. Another benefit of this combination was that the side effect profile of individual drugs was not significantly altered. We concluded that a combination of Bisphosphonates and Nitroglycerine is a rapid means to increase BMD without any additional side effects and hence is recommended in post-menopausal women with history of fractures.
\end{abstract}

Keywords: Osteoporosis, Nitroglycerine, BMD, Menopause, Fractures

\section{Introduction}

Osteoporosis represents a loss of skeletal bone mass and increased bone fragility. Many a times in our setup, the first time a patient is diagnosed, is with a fracture due to a trivial trauma. Majority of these patients are women in their fifties or sixties. As the average lifespan tends to increase and population of post-menopausal women tend to grow, this has reached a significant level with serious impact on health and economy ${ }^{1}$. A large number of drugs like calcium, vitamin D, bisphosphonates, Raloxifene(SERM) etc. are available for osteoporosis. NO (nitric oxide) is one such molecule which inhibits osteoclastic bone resorption in vitro and regulates bone remodelling ${ }^{2,3}$. It also promotes growth of Osteoblasts ${ }^{4}$. In vitro role of $\mathrm{NO}$ in prevention of osteoporosis comes from a study in which mice lacking NO synthase enzyme had defective bone formation due to defect in osteoblast functioning ${ }^{5}$. These factors suggest a role of Nitroglycerine in the management of osteoporosis.

Bone mineral density(BMD) is related to peak bone mass and subsequently bone loss; especially the T-score, which compares the patient's bone density with that of a control having peak bone mass. As per WHO, Osteoporosis is defined as $\mathrm{BMD} \geq 2.5 \mathrm{SD}$ ( standard deviation) below the normal mean of young adult woman i.e. T-Score $<2.5^{6}$.

In this study we will compare the effect of Nitroglycerine, Bisphosphonates, and Nitroglycerine and Bisphosphonates (combined) on BMD.

\section{Materials and Methods}

The study was carried in our hospital on 88 post-menopausal women who were randomised into three different groups based on computer generated random number table:
Group A: women on Alendronate 70 mg weekly

Group B: women on transdermal nitroglycerine $2 \%$.

Group C: women on the combination of Alendronate and transdermal nitroglycerine.

Since bisphosphonates are standard drugs for the management of osteoporosis, it was considered a standard group against which groups $\mathrm{B}$ and $\mathrm{C}$ were compared. All the women taking part in the study knew that they were a part of the study. However they did not know the group they belong to. In other words it was a single blinded study.

All the women taking part in the study were given 60 tablets of calcium $500 \mathrm{mg}$ and a single dose of Vitamin D 600000 IU at the start of study. Groups A, B, C had 30, 28, 30 patients respectively.

The inclusion criteria for the study were:

- healthy post-menopausal women with a hip BMD T score between 0 and -2.0

- age less than 60 years

- body mass index (BMI) of 20-30kg/m2

The exclusion criteria were:

- prior fractures;

- cardiac or coronary artery

- migraine

- metabolic bone disease

- any active malignancy

- corticosteroids or HRT use.

The primary outcome measure was BMD estimation at 12 months which was measured as a percentage of the baseline study at the start of study: 


\section{International Journal of Science and Research (IJSR) \\ ISSN (Online): 2319-7064}

Index Copernicus Value (2013): 6.14 | Impact Factor (2014): 5.611

[BMD (at 12 months) - BMD (at start)/BMD (at start)] $\mathbf{x} 100$

BMD was measured in the lumbar spine by dual-energy $\mathrm{X}$ ray absorptiometry (DXA) scanning at the beginning of the study, and again at 12 months. However all the patients were followed monthly and compliance checked by empty blister packs/ empty ointment tubes. During this period history of other drugs/ side effects was also recorded.

\section{Statistical Analysis}

Fisher's exact tests were used for categorical variables and t" tests and ANOVA test for continuous variables. Groups B and $\mathrm{C}$ were compared with Group A and various statistical analyses drawn.

Table 1: Baseline characteristics

\begin{tabular}{|c|c|c|c|}
\hline Characteristic & Group A & Group B & Group C \\
\hline Age & $53.26 \pm 5.37$ & $53.92 \pm 5.22$ & $53.06 \pm 5.03$ \\
\hline Total hip BMD (g/cm2) & $0.804 \pm 0.19$ & $0.752 \pm 0.20$ & $0.875 \pm 0.16$ \\
\hline BMI (kg/m2) & $26.0 \pm 3.5$ & $25.3 \pm 3.8$ & $26.88 \pm 3.0$ \\
\hline Smoking & 0 & 0 & 0 \\
\hline Age at menopause & $45.25 \pm 4.80$ & $46.05 \pm 4.5$ & $47.0 \pm 4.25$ \\
\hline
\end{tabular}

$p$ value $>0.05$ (ANOVA)

As stated in table 1 above, the baseline characters were similar in all the three groups ( $p>0.05$ ), the three groups were evaluated for compliance and side effects. All the patients were visited once weekly for the first month and later on monthly. During home visits their health status, activity level, work and other activities of daily activities were subjectively assessed. Fortunately none of the subjects developed any serious illness which would confound our study, and there were no drop outs. Finally at the end of 12 months bone marrow density was evaluated and expressed as a percentage of the baseline value.

The most common complaint of patients in group A was heart burn in 12 patients (FIGURE 1) which was corrected by sitting erect for up to 1 hour and drinking about $200 \mathrm{ml}$ of water post pill (ALENDRONATE) intake. Group B patients had headache in 6 patients (FIGURE 1); responded to a tablet of paracetamol $500 \mathrm{mg}$. Group C patients had headache in 8 patients and 14 patients had heart burn. Majority of these side effects were manifest in first month of study and none of the patients experienced any Side effects after third month onwards. This can be attributed to advise given to patients as stated above. This information can be depicted as below (Table 2 and Table 3):

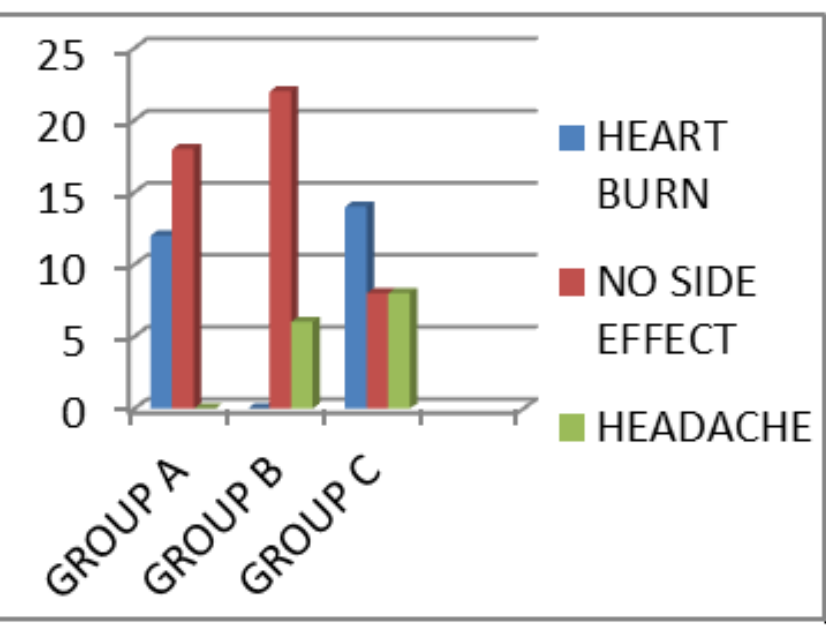

Figure 1: Side effect profile of three groups.

Table 2: Heart burn in groups A and C 1

\begin{tabular}{|c|c|c|c|}
\hline & Group A & Group C & Fischer Exact \\
\hline Heart Burn Present & 12 & 14 & $p>0.05$ \\
\hline Heart Burn Absent & 18 & 16 & \\
\hline
\end{tabular}

Table 3: Headache in groups B and C 1

\begin{tabular}{|c|c|c|c|}
\hline & Group B & Group C & Fischer Exact \\
\hline Headache Present & 6 & 8 & $P>0.05$ \\
\hline Headache Absent & 22 & 22 & \\
\hline
\end{tabular}

Bone mineral density was evaluated at 12 months and expressed as percentage change as shown below (FIGURE 2):

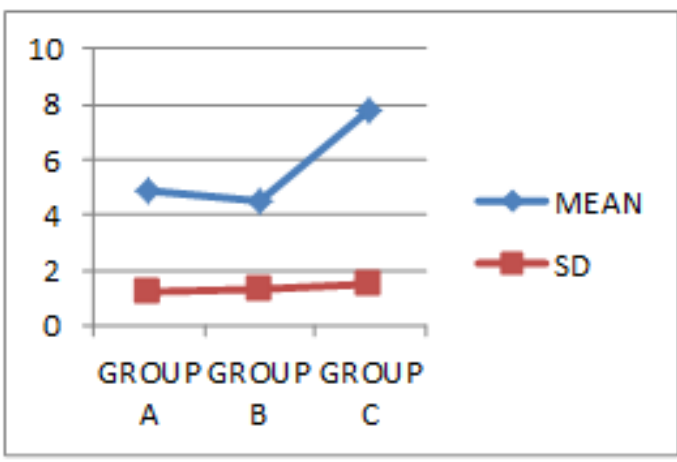

Figure 2: Percent increase in BMD at 1 Year

\section{Results}

On evaluating the above data and applying the various statistical tests, the following interpretations were made:

The most common side effect of Alendronate is heart burn ${ }^{7}$. On comparing the incidence of heart burn in Group A with Group C it was statistically insignificant $(p>0.05)$, meaning that addition of nitroglycerine did not influence the side effect profile of Alendronate. Sine there were no other side effects in both groups $\mathrm{A}$ and $\mathrm{C}$, it can be concluded that the combination is safe to use.

Headache is a common side effect of Nitroglycerine ${ }^{8}$. This side effect was observed in both groups B and C; intensity and frequency being more in first month of study possibly due to -Nitroglycerine tolerance" -----a phenomenon being observed after continuous Nitroglycerine usage. However, 


\section{International Journal of Science and Research (IJSR) \\ ISSN (Online): 2319-7064}

Index Copernicus Value (2013): 6.14 | Impact Factor (2014): 5.611

the incidence of headache in group $\mathrm{B}$ and group $\mathrm{C}$ was similar (and hence statistically insignificant; $p>0.05$ ). This implies that the combination with Alendronate didn't influence the side effect profile of individual drugs.

Hence this study points out that the combination of Alendronate with nitroglycerine is as safe as using individual drugs alone.

The percentage increase in group B was almost similar to group A suggesting that both Nitroglycerine and Alendronate are similar as for as their therapeutic efficacy is concerned $(p>0.05)$. However the percentage increase in BMD in group $C$ was statistically significant as compared to group A or group B $(p<0.01)$. Another benefit of this combination was that the side effect profile of individual drugs was not significantly altered.

\section{Discussion}

The incidence of osteoporosis is rising because of an increase in the elderly population throughout the world ${ }^{9,10}$. This is further compounded by the fact that the incidence of fractures increases with each passing year into post-menopausal life ${ }^{11}$. Most often such fractures are caused by trivial trauma which is very common in old age $\mathrm{e}^{12}$. Bisphosphonates continue to be used as a standard therapy for osteoporosis ${ }^{13}$. And they are also used as a standard therapy in prevention and treatment of fractures in post-menopausal women ${ }^{11,13}$. Although, in our study we found out that the combination of bisphosphonates and nitroglycerine is most effective for improving bone mineral density in post-menopausal women but the average increase at 12 months in BMD was $7.74 \%$ ( maximum increase was $10.01 \%$ ). This meagre increase in percentage (of BMD) does not justify its routine use in place of bisphosphonates which are time tested drugs for osteoporosis. However in case of fractures (due to trivial trauma) in post-menopausal women, increase in each percentage of BMD is important to prevent and treat fractures and thereby maintain a productive quality of life. The combination of bisphosphonates with nitroglycerine should be focussed on this group of patients till further studies become available, when they can be applied to all post-menopausal women.

\section{Conclusion}

It has been found from the above study that in postmenopausal women, at present, bisphosphonates alone are sufficient for routine treatment of osteoporosis but those with fractures (single or recurrent) should be offered a combination of Bisphosphonates and Nitroglycerine as it is a rapid means to treat the underlying defect of skeleton in a minimum amount of time without any additional side effects

\section{References}

[1] Majumdar SR, Lier DA, Beaupre LA, et al. -steoporosis case manager for patients with hip fractures: results of a cost-effective analysis conducted alongside a randomised tria”,. Arch Intern Med;169(1):25-31, 2009.
[2] MacIntyre I, Zaidi M, Towhidul ASM, Datta HK, Moonga BS, et al. Đsteoclast inhibition: An action of nitric oxide not mediated by cyclic GMP”, ProcNatl Acad Sci USA; 88:2936-2940, 1991.

[3] Chae HJ, Park RK, Chung HT, Kang JS, et al. Aitric oxide is a regulator of bone remodelling", J Pharm Pharmacol; 49:897-902, 1997.

[4] Brandi ML, Hukkanen M, Umeda T,et al. Bidirectional regulation of osteoclast function by nitric oxide synthase isoforms", Proc Natl Acad Sci USA; 92:2954-2958, 1995.

[5] Aguirre J, Buttery L, et al. Endothelial nitric oxide synthase gene deficient mice demonstrate marked retardation in post-natal bone formation, reduced bone volume, and defects in osteoblast maturation and activity”, Am J Pathol.;158:247-257, 2001

[6] Kanis JA. Assessment of fracture risk and its application to screening for post-menopausal osteoporosis: Synopsis of a WHO report. WHO study group”, Osteoporosis int.;4(6): 368-81, 1994.

[7] Liberman UA, Weiss SR, Broll J, Minne HW, Quan H, Bell NH, Rodriguez-Portales J, et al. Effect of oral alendronate on bone mineral density and the incidence of fractures in postmenopausal osteoporosis. The Alendronate Phase III Osteoporosis Treatment Study Group", N Engl J Med; 333: 1437-1443,1995.

[8] Riegel B, Heywood G, Jackson W, Kennedy A . Effect of nitroglycerin ointment placement on the severity of headache and flushing in patients with cardiac disease", Heart Lung ; 17:426-431, 1988.

[9] Rosen CJ, Tenenhouse A. - Biochemical markers of bone turnover - a look at laboratory tests that reflect bone status", Postgrad Med; 104:101-112, 1998.

[10] Riggs BL, Melton LJ. The prevention and treatment of osteoporosis", N Engl. J Med ; 327:620-627, 1992.

[11] Nilas L, Christiansen C. Rates of bone loss in normal women: Evidence of accelerated trabecular bone loss after the menopause", Eur J Clin. Invest; 18:529-534, 1998.

[12] Greenblatt D. Treatment of postmenopausal osteoporosis". Pharmacotherapy; 25 (4) ,574-584, 2005.

[13] Wimalawansa S J. Aitroglycerin Therapy Is as Efficacious as Standard Estrogen Replacement Therapy (Premarin) in Prevention of Oophorectomy-Induced Bone Loss: A Human Pilot Clinical Study," JOURNAL OF BONE AND MINERAL RESEARCH; 15(11):22402244, 2000.

\section{Author Profile}

Raheeb Ahmaad Shah did his MBBS and MS in surgery from GMC Srinagar. He has authored three papers on various surgical specialties. He is presently working as a medical officer in National Health Mission. 